\title{
ON INTEGRAL INEQUALITIES OF HERMITE-HADAMARD TYPE FOR COORDINATED $r$-MEAN CONVEX FUNCTIONS
}

\author{
DAN-DAN GAO, BO-YAN XI, YING WU, AND BAI-NI GUO
}

Received 24 January, 2019

\begin{abstract}
In the paper, the authors first introduce a concept " $r$-mean convex function on coordinates" and then establish several integral inequalities of the Hermite-Hadamard type for $r$-convex functions and $r$-mean convex functions on coordinates.
\end{abstract}

2010 Mathematics Subject Classification: 26D15; 26A51; 26E60; 41A55

Keywords: inequality of the Hermite-Hadamard type, $r$-convex function, coordinated $r$-mean convex function

\section{INTRODUCTION}

The following definition is well known in the literature.

Definition 1. A function $h: I \subseteq \mathbb{R} \rightarrow \mathbb{R}$ is called to be convex if the inequality

$$
h(\lambda x+(1-\lambda) y) \leq \lambda h(x)+(1-\lambda) h(y)
$$

holds for all $x, y \in I$ and $\lambda \in[0,1]$. If the above inequality is reversed, then we call $f$ a concave function.

If $h: I \subseteq \mathbb{R} \rightarrow \mathbb{R}$ is a convex function on $[a, b]$ with $a, b \in I$ and $a<b$, then

$$
h\left(\frac{a+b}{2}\right) \leq \frac{1}{b-a} \int_{a}^{b} h(t) \mathrm{d} t \leq \frac{h(a)+h(b)}{2} .
$$

This inequality is called Hermite-Hardamard's integral inequality in the literature.

Hermite-Hadamard's integral inequality (1.1) has been refined, generalized, and applied by a number of mathematicians. For more information, please refer to $[7,12$, 25], for example.

Definition 2 ([2,3]). A real function $h$ defined on a convex set $\mathbb{D} \subseteq \mathbb{R}^{n}$ is said to be $r$-convex if

$$
\phi\left(q_{1} x+q_{2} y\right) \leq \begin{cases}\ln \left(q_{1} e^{r h(x)}+q_{2} e^{r h(y)}\right)^{1 / r}, & r \neq 0 \\ q_{1} h(x)+q_{2} h(y), & r=0\end{cases}
$$

for all $x, y \in \mathbb{D}$ and $q_{1}, q_{2} \geq 0$ with $q_{1}+q_{2}=1$. 
Definition 3 ([14]). For $r \in \mathbb{R}$, a function $h: I \subseteq \mathbb{R} \rightarrow \mathbb{R}_{+}=(0, \infty)$ is called to be $r$-convex if

$$
h(\lambda x+(1-\lambda) y) \leq \begin{cases}\left\{\lambda[h(x)]^{r}+(1-\lambda)[h(y)]^{r}\right\}^{1 / r}, & r \neq 0 \\ {[h(x)]^{\lambda}[h(y)]^{1-\lambda},} & r=0\end{cases}
$$

holds for all $x, y \in I$ and $\lambda \in[0,1]$. If the inequality (1.2) is reversed, then we call $h$ an $r$-concave function.

Definition 4 ([22,24]). For $r \in \mathbb{R}$, a function $h: I \subseteq \mathbb{R}_{+} \rightarrow \mathbb{R}_{+}$is called to be $r$-mean convex if

$$
h\left(\left[\lambda x^{r}+(1-\lambda) y^{r}\right]^{1 / r}\right) \leq\left\{\lambda[h(x)]^{r}+(1-\lambda)[h(y)]^{r}\right\}^{1 / r}, \quad r \neq 0
$$

and

$$
h\left(x^{\lambda} y^{1-\lambda}\right) \leq[h(x)]^{\lambda}[h(y)]^{1-\lambda}, \quad r=0
$$

hold for $x, y \in I$ and $\lambda \in[0,1]$. If the above inequality is reversed, then we call $h$ an $r$-mean concave function.

Definition 5 ([27]). For $r \in \mathbb{R}$, a function $h: I \subseteq \mathbb{R}_{+} \rightarrow \mathbb{R}_{+}$is called to be geometrically $r$-convex if

$$
h\left(x^{\lambda} y^{1-\lambda}\right) \leq \begin{cases}\left\{\lambda[h(x)]^{r}+(1-\lambda)[h(y)]^{r}\right\}^{1 / r}, & r \neq 0 \\ {[h(x)]^{\lambda}[h(y)]^{1-\lambda},} & r=0\end{cases}
$$

holds for $x, y \in I$ and $\lambda \in[0,1]$.

For properties and inequalities of the Hermite-Hadamard type relating to $r$-convex functions, $r$-mean convex functions, and geometrically $r$-convex functions, please read the papers $[8,11,14,17,18,21,22,24,27,29,30]$ and closely related references.

Definition 6 ([5,7]). A function $h: \Delta=[a, b] \times[c, d] \subseteq \mathbb{R}^{2} \rightarrow \mathbb{R}$ is called to be convex on coordinates on $\Delta$ with $a<b$ and $c<d$ if the partial functions

$$
h_{y}:[a, b] \rightarrow \mathbb{R}, \quad h_{y}(u)=h(u, y) \quad \text { and } \quad h_{x}:[c, d] \rightarrow \mathbb{R}, \quad h_{x}(v)=h(x, v)
$$

are convex for all $x \in[a, b]$ and $y \in[c, d]$.

Definition 7 ([5,7]). A function $h: \Delta=[a, b] \times[c, d] \subseteq \mathbb{R}^{2} \rightarrow \mathbb{R}$ is called to be convex on coordinates on $\Delta$ with $a<b$ and $c<d$ if the inequality

$$
\begin{aligned}
h(t x+ & (1-t) z, \lambda y+(1-\lambda) w) \\
& \leq t \lambda h(x, y)+t(1-\lambda) h(x, w)+(1-t) \lambda h(z, y)+(1-t)(1-\lambda) h(z, w)
\end{aligned}
$$

holds for all $t, \lambda \in[0,1]$ and $(x, y),(z, w) \in \Delta$.

Definition 8 ([1]). A function $h: \Delta=[a, b] \times[c, d] \subseteq \mathbb{R}^{2} \rightarrow \mathbb{R}_{+}$is called coordinated logarithmically convex on $\Delta$ with $a<b$ and $c<d$ for all $t, \lambda \in[0,1]$ and $(x, y),(z, w) \in \Delta$ if 


$$
\begin{aligned}
h(t x+(1-t) z, & \lambda y+(1-\lambda) w) \\
& \leq[h(x, y)]^{t \lambda}[h(x, w)]^{t(1-\lambda)}[h(z, y)]^{(1-t) \lambda}[h(z, w)]^{(1-t)(1-\lambda)} .
\end{aligned}
$$

Definition 9 ([13]). For $r \in \mathbb{R}$, a function $h: \Delta=[a, b] \times[c, d] \subseteq \mathbb{R}^{2} \rightarrow \mathbb{R}_{+}$is called coordinated $r$-convex on $\Delta$ with $a<b$ and $c<d$ for all $t, \lambda \in[0,1]$ and $(x, y),(z, w) \in \Delta$ if

$$
\begin{aligned}
h(t x & +(1-t) z, \lambda y+(1-\lambda) w) \\
& \leq\left\{\begin{array}{cc}
\left\{t \lambda[h(x, y)]^{r}+t(1-\lambda)[h(x, w)]^{r}+(1-t) \lambda[h(z, y)]^{r}\right. & r \neq 0 \\
\left.+(1-t)(1-\lambda)[h(z, w)]^{r}\right\}^{1 / r}, & \\
{[h(x, y)]^{t \lambda}[h(x, w)]^{t(1-\lambda)}[h(z, y)]^{(1-t) \lambda}[h(z, w)]^{(1-t)(1-\lambda)},} & r=0 .
\end{array}\right.
\end{aligned}
$$

Remark 1. Obviously, if putting $r=0$ in Definition 9, then $h$ is just the ordinary coordinated logarithmically convex function on $\Delta$.

Stolarsky's mean $E(u, v ; r, s)$ for $(u, v ; r, s) \in \mathbb{R}_{+}^{2} \times \mathbb{R}^{2}$ is defined by

$$
\begin{aligned}
E(u, v ; r, s) & =\left[\frac{r\left(v^{s}-u^{s}\right)}{s\left(v^{r}-u^{r}\right)}\right]^{1 /(s-r)}, & r s(r-s)(u-v) & \neq 0 ; \\
E(u, v ; 0, s) & =\left[\frac{v^{s}-u^{s}}{s(\ln v-\ln u)}\right]^{1 / s}, & s(u-v) & \neq 0 ; \\
E(u, s ; r, r) & =\frac{1}{e^{1 / r}}\left(\frac{u^{u^{r}}}{v^{v^{r}}}\right)^{1 /\left(u^{r}-v^{r}\right)}, & r(u-v) & \neq 0 ; \\
E(u, v ; 0,0) & =\sqrt{u v}, & u & \neq v ; \\
E(u, u ; r, s) & =u, & u & =v .
\end{aligned}
$$

The quantities $L(u, v)=E(u, v ; 0,1)$ and $L_{r}(u, v)=E(u, v ; r, r+1)$ are respectively called the logarithmic mean and generalized logarithmic mean of two real positive numbers $u, v$. For more information on Stolarsky's mean, please refer to the papers $[9,10,15,16,19,20]$ and closely related references therein.

Theorem 1 ([8, Theorem 2.1]). Suppose that $h:[a, b] \subseteq \mathbb{R} \rightarrow \mathbb{R}_{+}$is a logarithmically convex function with $a<b$. Then

$$
\frac{1}{b-a} \int_{a}^{b} h(t) \mathrm{d} t \leq L(h(a), h(b)),
$$

where $L(x, y)$ is the logarithmic mean.

Theorem 2 ([8, Theorem 3.1]). Suppose that $h:[a, b] \subseteq \mathbb{R} \rightarrow \mathbb{R}_{+}$is an $r$-convex function for $r \in \mathbb{R}$ with $a<b$. Then

$$
\frac{1}{b-a} \int_{a}^{b} h(t) \mathrm{d} t \leq L_{r}(h(a), h(b)),
$$


where $L_{r}(x, y)$ is the generalized logarithmic mean.

Theorem $3([5,7])$. Let $h: \Delta=[a, b] \times[c, d] \subseteq \mathbb{R}^{2}$ be convex on coordinates on $\Delta$ with $a<b$ and $c<d$. Then

$$
\begin{aligned}
& h\left(\frac{a+b}{2}, \frac{c+d}{2}\right) \leq \frac{1}{2}\left[\frac{1}{b-a} \int_{a}^{b} h\left(x, \frac{c+d}{2}\right) \mathrm{d} x+\frac{1}{d-c} \int_{c}^{d} h\left(\frac{a+b}{2}, y\right) \mathrm{d} y\right] \\
& \quad \leq \frac{1}{(b-a)(d-c)} \int_{a}^{b} \int_{c}^{d} h(x, y) \mathrm{d} y \mathrm{~d} x \\
& \quad \leq \frac{1}{4}\left[\frac{1}{b-a} \int_{a}^{b}[h(x, c)+h(x, d)] \mathrm{d} x+\frac{1}{d-c} \int_{c}^{d}[h(a, y)+h(b, y)] \mathrm{d} y\right] \\
& \quad \leq \frac{1}{4}[h(a, c)+h(b, c)+h(a, d)+h(b, d)] .
\end{aligned}
$$

Theorem 4 ([1, Theorem 3.3]). Suppose that $h: \Delta=[a, b] \times[c, d] \subseteq \mathbb{R}^{2} \rightarrow \mathbb{R}_{+}$ is logarithmically convex on coordinates $\Delta$ for $a<b$ and $c<d$. Let

$$
A=\frac{h(a, c) h(b, d)}{h(b, c) h(a, d)}, \quad B=\frac{h(a, d)}{h(b, d)}, \quad C=\frac{h(b, c)}{h(b, d)} .
$$

Then

where

$$
\frac{1}{(b-a)(d-c)} \int_{a}^{b} \int_{c}^{d} h(x, y) \mathrm{d} y \mathrm{~d} x \leq M_{h}(\Delta),
$$

$$
\begin{aligned}
M_{h}(\Delta)= & \begin{array}{ll}
1, & A=B=C=1 ; \\
\frac{B-1}{\ln B} \frac{C-1}{\ln C}, & A=1 ; \\
H(C), & C=1 ; \\
H(B), & A=B=1 ; \\
\frac{C-1}{\ln C}, & A=C=1 ; \\
\frac{B-1}{\ln B}, & B=C=1 ; \\
\frac{\gamma+\ln (-\ln A)+E i(1,-\ln A)}{\ln A}, & B, B, C>0 ; \\
\frac{1}{2}\left[\frac{B-1}{\ln B}+\frac{A B-1}{\ln (A B)}\right], & \text { otherwise, } \\
\int_{0}^{1} C^{\beta} \frac{A B-1}{\ln (A B)} \mathrm{d} \beta, & \frac{E i(1,-\ln A)+\ln \ln x-\text { Ei }(1,-\ln (\text { Ax }))-\ln \ln (\text { Ax })}{\ln A}
\end{array} \\
H(x)= & + \begin{cases}\frac{2 \ln (\ln A)-\ln (-\ln A)}{\ln A}, & -1<\frac{\ln x}{\ln A}<0 ; \\
0, & \text { otherwise },\end{cases} \\
\text { Ei }(x)= & V \cdot P \cdot \int_{-x}^{\infty} \frac{e^{-t} \mathrm{~d} t,}{t}
\end{aligned}
$$


is the exponential integral function, and $\gamma$ is the Euler constant.

In very recent years, some other kinds of inequalities of the Hermite-Hadamard type were created in, for example, $[1,4,6,13,23,26,28,30]$ and closely related references therein.

In this paper, by combining the definition of convex functions with the definition of coordinated convex functions, we introduce the concept " $r$-mean convex function on coordinates" and establish integral inequalities of the Hermite-Hadamard type for $r$-mean convex functions on coordinates.

\section{A DEFINITION AND A LEMMA}

In this section, we define a concept " $r$-mean convex function on coordinates" and prepare a lemma necessary for establishing new inequalities of the HermiteHadamard type for $r$-mean convex function on coordinates.

Definition 10. For $r \in \mathbb{R}$, a function $h: \Delta=[a, b] \times[c, d] \subseteq \mathbb{R}_{+}^{2} \rightarrow \mathbb{R}_{+}$is called a coordinated $r$-mean convex on $\Delta$ with $a<b$ and $c<d$ if

$$
\begin{aligned}
h\left(\left[t x^{r}+\right.\right. & \left.\left.(1-t) z^{r}\right]^{1 / r},\left[\lambda y^{r}+(1-\lambda) w^{r}\right]^{1 / r}\right) \leq\left\{t \lambda[h(x, y)]^{r}\right. \\
& \left.+t(1-\lambda)[h(x, w)]^{r}+(1-t) \lambda[h(z, y)]^{r}+(1-t)(1-\lambda)[h(z, w)]^{r}\right\}^{1 / r}
\end{aligned}
$$

for $r \neq 0$ and

$$
h\left(x^{t} z^{1-t}, y^{\lambda} w^{1-\lambda}\right) \leq[h(x, y)]^{t \lambda}[h(x, w)]^{t(1-\lambda)}[h(z, y)]^{(1-t) \lambda}[h(z, w)]^{(1-t)(1-\lambda)}
$$

for $r=0$, where $t, \lambda \in[0,1]$ and $(x, y),(z, w) \in \Delta$.

Remark 2. In Definition 10, if $r=0$, then we call $h$ a coordinated geometrically convex function on $\Delta$; if $r=-1$, then we call $f$ a coordinated harmonically convex function on $\Delta$.

In order to prove our main theorems, we need the following lemma.

Lemma 1. Let $r, R, T, S \in \mathbb{R}$ and $r \neq 0$ such that $R+T+S>0, R+S>0$, $T+S>0$, and $S>0$. Then

$$
F(R, T, S, r) \triangleq \int_{0}^{1} \int_{0}^{1}(R t+T \lambda+S)^{1 / r} \mathrm{~d} \lambda \mathrm{d} t
$$




$$
=\left\{\begin{array}{l}
r^{2}\left[(R+T+S)^{1 / r+2}-(R+S)^{1 / r+2}\right. \\
\frac{\left.-(T+S)^{1 / r+2}+S^{1 / r+2}\right]}{(r+1)(2 r+1) R T}, \quad(r+1)\left(r+\frac{1}{2}\right) R T \neq 0 ; \\
\frac{r\left[(R+T+S)^{1 / r+1}-S^{1 / r+1}\right]}{(r+1)(R+T)}, \quad(r+1)(R+T) \neq 0, \quad R T=0 ; \\
\frac{-(T+S) \ln (T+S)+S \ln S}{R T}, \quad r=-1, \quad R T \neq 0 ; \\
\frac{\ln (R+T+S)-\ln S}{R+T}, \quad r=-1, \quad R T=0, \quad R+T \neq 0 ; \\
\frac{\ln (R+S)+\ln (T+S)-\ln (R+T+S)-\ln S}{R T}, \quad r=-\frac{1}{2}, \quad R T \neq 0 ; \\
\frac{1}{S(R+T+S)}, \quad r=-\frac{1}{2}, \quad R T=0 ; \\
S^{1 / r}, \quad r \neq 0, \quad R=T=0 .
\end{array}\right.
$$

Proof. This follows from a straightforward computation.

\section{MAIN RESULTS}

In this section, we establish some integral inequalities of the Hermite-Hadamard type for $r$-mean convex functions on coordinates.

Theorem 5. Suppose that $h:[a, b] \subseteq \mathbb{R}_{+} \rightarrow \mathbb{R}_{+}$is an $r$-mean convex function on $[a, b]$ for all $r \in \mathbb{R}$ with $r \neq 0$. If $h \in L_{1}[a, b]$, then

$$
h\left(\left(\frac{a^{r}+b^{r}}{2}\right)^{1 / r}\right) \leq\left(\frac{r}{b^{r}-a^{r}} \int_{a}^{b} \frac{[h(x)]^{r}}{x^{1-r}} \mathrm{~d} x\right)^{1 / r}
$$

and

$$
\frac{r}{b^{r}-a^{r}} \int_{a}^{b} \frac{h(x)}{x^{1-r}} \mathrm{~d} x \leq L_{r}(h(a), h(b)),
$$

where $L_{r}(x, y)$ is the generalized logarithmic mean.

Proof. For $t \in[0,1]$, by the $r$-mean convexity of $h$ on $[a, b]$, we have

$$
\begin{aligned}
& h\left(\left(\frac{a^{r}+b^{r}}{2}\right)^{1 / r}\right)=h\left(\left[\frac{t a^{r}+(1-t) b^{r}+(1-t) a^{r}+t b^{r}}{2}\right]^{1 / r}\right) \\
& \leq\left(\frac{1}{2}\right)^{1 / r}\left\{\left[h\left(\left[t a^{r}+(1-t) b^{r}\right]^{1 / r}\right)\right]^{r}+\left[h\left(\left[(1-t) a^{r}+t b^{r}\right]^{1 / r}\right)\right]^{r}\right\}^{1 / r}
\end{aligned}
$$

If $r>0$, then

$$
\begin{aligned}
& {\left[h\left(\left(\frac{a^{r}+b^{r}}{2}\right)^{1 / r}\right)\right]^{r}} \\
& \leq \frac{1}{2}\left\{\left[h\left(\left[t a^{r}+(1-t) b^{r}\right]^{1 / r}\right)\right]^{r}+\left[h\left(\left[(1-t) a^{r}+t b^{r}\right]^{1 / r}\right)\right]^{r}\right\} .
\end{aligned}
$$


Integrating with respect to $t$ over $[0,1]$ and putting $x=t a^{r}+(1-t) b^{r}$ for $t \in[0,1]$ yield

$$
h\left(\left(\frac{a^{r}+b^{r}}{2}\right)^{1 / r}\right) \leq\left(\frac{r}{b^{r}-a^{r}} \int_{a}^{b} \frac{[h(x)]^{r}}{x^{1-r}} \mathrm{~d} x\right)^{1 / r} .
$$

Similarly, if $r<0$, then we obtain the inequality (3.1). Taking $x^{r}=t a^{r}+(1-t) b^{r}$ for $t \in[0,1]$ and utilizing the $r$-mean convexity of $f$ on $[a, b]$ lead to

$$
\begin{aligned}
\frac{r}{b^{r}-a^{r}} \int_{a}^{b} \frac{h(x)}{x^{1-r}} \mathrm{~d} x & =\int_{0}^{1} h\left(\left[t a^{r}+(1-t) b^{r}\right]^{1 / r}\right) \mathrm{d} t \\
& \leq \int_{0}^{1}\left\{t[h(a)]^{r}+(1-t)[h(b)]^{r}\right\}^{1 / r} \mathrm{~d} t=L_{r}(h(a), h(b)) .
\end{aligned}
$$

Theorem 5 is thus proved.

Theorem 6. Let $h: \Delta=[a, b] \times[c, d] \subseteq \mathbb{R}_{+}^{2} \rightarrow \mathbb{R}_{+}$and let $a<b, r \in \mathbb{R}$ with $r \neq 0$, and $h \in L_{1}(\Delta)$. If $h$ is a coordinated $r$-mean convex function on $\Delta$, then

$$
\begin{aligned}
h\left(\left(\frac{a^{r}+b^{r}}{2}\right)^{1 / r},\left(\frac{c^{r}+d^{r}}{2}\right)^{1 / r}\right) & \\
\leq & {\left[\frac{r^{2}}{\left(b^{r}-a^{r}\right)\left(d^{r}-c^{r}\right)} \int_{a}^{b} \int_{c}^{d} \frac{[h(x, y)]^{r}}{(x y)^{1-r}} \mathrm{~d} y \mathrm{~d} x\right]^{1 / r} }
\end{aligned}
$$

and

$$
\begin{aligned}
\frac{r^{2}}{\left(b^{r}-a^{r}\right)\left(d^{r}-c^{r}\right)} \int_{a}^{b} \int_{c}^{d} \frac{h(x, y)}{(x y)^{1-r} \mathrm{~d} y \mathrm{~d} x} & r>0 ; \\
\leq & \begin{array}{ll}
\frac{F(R, T, S, r)}{2^{1 / r}}, & \\
\min \left\{L_{r}(h(a, c), h(a, d))+L_{r}(h(b, c), h(b, d)),\right. & \\
\frac{\left.L_{r}(h(a, c), h(b, c))+L_{r}(h(a, d), h(b, d))\right\}}{2}, & r<0,
\end{array}
\end{aligned}
$$

where $L_{r}(x, y)$ is the generalized logarithmic mean, $M_{h}(\Delta)$ and $F(R, T, S, r)$ are respectively defined as in (1.3) and Lemma 1 , and

$$
\begin{aligned}
& R=h^{r}(a, c)+h^{r}(a, d)-h^{r}(b, c)-h^{r}(b, d), \\
& \left.T=h^{r}(a, c)+h^{r}(b, c)-h(a, d)\right]^{r}-h^{r}(b, d), \\
& S=h^{r}(b, c)+h^{r}(a, d)+2 h^{r}(b, d) .
\end{aligned}
$$

Proof. From the coordinated $r$-mean convexity of $h$ on $\Delta$, we have

$$
\begin{aligned}
h\left(\left(\frac{a^{r}+b^{r}}{2}\right)^{1 / r}\right. & \left.,\left(\frac{c^{r}+d^{r}}{2}\right)^{1 / r}\right)=h\left(\left[\frac{t a^{r}+(1-t) b^{r}+(1-t) a^{r}+t b^{r}}{2}\right]^{1 / r},\right. \\
& {\left.\left[\frac{\lambda c^{r}+(1-\lambda) d^{r}+(1-\lambda) c^{r}+\lambda d^{r}}{2}\right]^{1 / r}\right) }
\end{aligned}
$$




$$
\begin{aligned}
\leq & \left(\frac{1}{4}\right)^{1 / r}\left\{\left[h\left(\left[t a^{r}+(1-t) b^{r}\right]^{1 / r},\left[\lambda c^{r}+(1-\lambda) d^{r}\right]^{1 / r}\right)\right]^{r}\right. \\
& +\left[h\left(\left[t a^{r}+(1-t) b^{r}\right]^{1 / r},\left[(1-\lambda) c^{r}+\lambda d^{r}\right]^{1 / r}\right)\right]^{r} \\
& +\left[h\left(\left[(1-t) a^{r}+t b^{r}\right]^{1 / r},\left[\lambda c^{r}+(1-\lambda) d^{r}\right]^{1 / r}\right)\right]^{r} \\
& \left.+\left[h\left(\left[(1-t) a^{r}+t b^{r}\right]^{1 / r},\left[(1-\lambda) c^{r}+\lambda d^{r}\right]^{1 / r}\right)\right]^{r}\right\}^{1 / r}
\end{aligned}
$$

for all $t, \lambda \in[0,1]$. As did in the proof of the inequality (3.2), we can obtain

$$
h\left(\left[\frac{a^{r}+b^{r}}{2}\right]^{1 / r},\left[\frac{c^{r}+d^{r}}{2}\right]^{1 / r}\right) \leq\left[\frac{r^{2}}{\left(b^{r}-a^{r}\right)\left(d^{r}-c^{r}\right)} \int_{a}^{b} \int_{c}^{d} \frac{[h(x, y)]^{r}}{(x y)^{1-r}} \mathrm{~d} y \mathrm{~d} x\right]^{1 / r} .
$$

Letting $x^{r}=t a^{r}+(1-t) b^{r}$ and $y^{r}=t c^{r}+(1-t) d^{r}$ for $t, \lambda \in[0,1]$ and using the coordinated $r$-mean convexity of $h$ on $\Delta$ lead to

$$
\begin{gathered}
\frac{r^{2}}{\left(b^{r}-a^{r}\right)\left(d^{r}-c^{r}\right)} \int_{a}^{b} \int_{c}^{d} \frac{h(x, y)}{(x y)^{1-r}} \mathrm{~d} y \mathrm{~d} x=\int_{0}^{1} \int_{0}^{1} h\left(\left[t a^{r}+(1-t) b^{r}\right]^{1 / r},\right. \\
\left.\left[\lambda c^{r}+(1-\lambda) d^{r}\right]^{1 / r}\right) \mathrm{d} t \mathrm{~d} \lambda \leq \int_{0}^{1} \int_{0}^{1}\left\{t \lambda h^{r}(a, c)+t(1-\lambda) h^{r}(a, d)\right. \\
\left.+(1-t) \lambda h^{r}(b, c)+(1-t)(1-\lambda) h^{r}(b, d)\right\}^{1 / r} \mathrm{~d} t \mathrm{~d} \lambda .
\end{gathered}
$$

(1) When $r>0$, since $2 \lambda t \leq \lambda+t$ for all $\lambda, t \in[0,1]$, by Lemma 1 and the inequality (3.3), we have

$$
\begin{gathered}
\frac{r^{2}}{\left(b^{r}-a^{r}\right)\left(d^{r}-c^{r}\right)} \int_{a}^{b} \int_{c}^{d} \frac{h(x, y)}{(x y)^{1-r}} \mathrm{~d} y \mathrm{~d} x \leq \int_{0}^{1} \int_{0}^{1}\left\{t \lambda h^{r}(a, c)\right. \\
\left.+t(1-\lambda) h^{r}(a, d)+(1-t) \lambda h^{r}(b, c)+(1-t)(1-\lambda) h^{r}(b, d)\right\}^{1 / r} \mathrm{~d} t \mathrm{~d} \lambda \\
\leq \frac{1}{2^{1 / r}} \int_{0}^{1} \int_{0}^{1}\left\{(t+\lambda) h^{r}(a, c)+(t+(1-\lambda)) h^{r}(a, d)+((1-t)+\lambda) h^{r}(b, c)\right. \\
\left.+((1-t)+(1-\lambda)) h^{r}(b, d)\right\}^{1 / r} \mathrm{~d} t \mathrm{~d} \lambda=\frac{1}{2^{1 / r}} F(R, T, S, r) .
\end{gathered}
$$

(2) When $r<0$, for $x, y>0$, using the convexity of $g(\lambda)=[\lambda x+(1-\lambda) y]^{1 / r}$ on $[0,1]$ and by the inequality $(3.3)$, we obtain

$$
\begin{aligned}
& \frac{r^{2}}{\left(b^{r}-a^{r}\right)\left(d^{r}-c^{r}\right)} \int_{a}^{b} \int_{c}^{d} \frac{h(x, y)}{(x y)^{1-r}} \mathrm{~d} y \mathrm{~d} x \leq \int_{0}^{1} \int_{0}^{1}\left\{t \left[\lambda h^{r}(a, c)\right.\right. \\
& \left.\left.+(1-\lambda) h^{r}(a, d)\right]+(1-t)\left[\lambda h^{r}(b, c)+(1-\lambda) h^{r}(b, d)\right]\right\}^{1 / r} \mathrm{~d} t \mathrm{~d} \lambda \\
& \leq \int_{0}^{1} \int_{0}^{1}\left\{t\left[\lambda h^{r}(a, c)+(1-\lambda) h^{r}(a, d)\right]^{1 / r}+(1-t)\left[\lambda h^{r}(b, c)\right.\right.
\end{aligned}
$$




$$
\begin{gathered}
\left.\left.+(1-\lambda) h^{r}(b, d)\right]^{1 / r}\right\} \mathrm{d} t \mathrm{~d} \lambda=\frac{1}{2} \int_{0}^{1}\left[\left\{\lambda h^{r}(a, c)+(1-\lambda) h^{r}(a, d)\right\}^{1 / r}\right. \\
\left.+\left\{\lambda h^{r}(b, c)+(1-\lambda) h^{r}(b, d)\right\}^{1 / r}\right] \mathrm{d} \lambda \\
=\frac{L_{r}(h(a, c), h(a, d))+L_{r}(h(b, c), h(b, d))}{2} .
\end{gathered}
$$

Similarly, we have

$$
\begin{gathered}
\frac{r^{2}}{\left(b^{r}-a^{r}\right)\left(d^{r}-c^{r}\right)} \int_{a}^{b} \int_{c}^{d} \frac{h(x, y)}{(x y)^{1-r}} \mathrm{~d} y \mathrm{~d} x \leq \int_{0}^{1} \int_{0}^{1}\left\{\lambda \left[t h^{r}(a, c)\right.\right. \\
\left.\left.+(1-t) h^{r}(b, c)\right]+(1-\lambda)\left[t h^{r}(a, d)+(1-t) h^{r}(b, d)\right]\right\}^{1 / r} \mathrm{~d} t \mathrm{~d} \lambda \\
\leq \frac{L_{r}(h(a, c), h(b, c))+L_{r}(h(a, d), h(b, d))}{2} .
\end{gathered}
$$

Theorem 6 is thus proved.

Theorem 7. Suppose that $h: \Delta=[a, b] \times[c, d] \subseteq \mathbb{R}_{+}^{2} \rightarrow \mathbb{R}_{+}$is geometrically convex on coordinates $\Delta$ for $a<b$ and $c<d$. Then

$$
h(\sqrt{a b}, \sqrt{c d}) \leq \frac{1}{(\ln b-\ln a)(\ln d-\ln c)} \int_{a}^{b} \int_{c}^{d} \frac{h(x, y)}{x y} \mathrm{~d} y \mathrm{~d} x \leq M_{h}(\Delta),
$$

where $M_{h}(\Delta)$ is defined as in (1.3).

Proof. Since $h$ is geometrically convex on coordinates $\Delta$, we have

$$
\begin{aligned}
& h(\sqrt{a b}, \sqrt{c d})=\int_{0}^{1} \int_{0}^{1} h\left(\left(a^{t} b^{1-t}\right)^{1 / 2}\left(a^{1-t} b^{t}\right)^{1 / 2},\right. \\
& \left.\left(c^{\lambda} d^{1-\lambda}\right)^{1 / 2}\left(c^{1-\lambda} d^{\lambda}\right)^{1 / 2}\right) \mathrm{d} t \mathrm{~d} \lambda \\
& \leq \int_{0}^{1} \int_{0}^{1}\left[h\left(a^{t} b^{1-t}, c^{\lambda} d^{1-\lambda}\right) h\left(a^{t} b^{1-t}, c^{1-\lambda} d^{\lambda}\right)\right. \\
& \left.\times h\left(a^{1-t} b^{t}, c^{\lambda} d^{1-\lambda}\right) h\left(a^{1-t} b^{t}, c^{1-\lambda} d^{\lambda}\right)\right]^{1 / 4} \mathrm{~d} t \mathrm{~d} \lambda \\
& \leq \frac{1}{4} \int_{0}^{1} \int_{0}^{1}\left[h\left(a^{t} b^{1-t}, c^{\lambda} d^{1-\lambda}\right)+h\left(a^{t} b^{1-t}, c^{1-\lambda} d^{\lambda}\right)\right. \\
& \left.+h\left(a^{1-t} b^{t}, c^{\lambda} d^{1-\lambda}\right)+h\left(a^{1-t} b^{t}, c^{1-\lambda} d^{\lambda}\right)\right] \mathrm{d} t \mathrm{~d} \lambda \\
& =\frac{1}{(\ln b-\ln a)(\ln d-\ln c)} \int_{a}^{b} \int_{c}^{d} \frac{h(x, y)}{x y} \mathrm{~d} y \mathrm{~d} x .
\end{aligned}
$$

Putting $x=a^{t} b^{1-t}$ and $y=c^{\lambda} d^{1-\lambda}$ for $0 \leq t$ and $\lambda \leq 1$, utilizing the coordinated geometric convexity of $h$, and employing Theorem 4 result in

$$
\frac{1}{(\ln b-\ln a)(\ln d-\ln c)} \int_{a}^{b} \int_{c}^{d} \frac{h(x, y)}{x y} \mathrm{~d} y \mathrm{~d} x
$$




$$
\begin{gathered}
=\int_{0}^{1} \int_{0}^{1} h\left(a^{t} b^{1-t}, c^{\lambda} d^{1-\lambda}\right) \mathrm{d} \lambda \mathrm{d} t \\
\leq \int_{0}^{1} \int_{0}^{1}[h(a, c)]^{t \lambda}[h(a, d)]^{t(1-\lambda)}[h(b, c)]^{(1-t) \lambda}[h(b, d)]^{(1-t)(1-\lambda)} \mathrm{d} \lambda \mathrm{d} t \\
=M_{h}(\Delta) .
\end{gathered}
$$

Theorem 7 is thus proved.

Corollary 1. Suppose that $h: \Delta=[a, b] \times[c, d] \subseteq \mathbb{R}_{+}^{2} \rightarrow \mathbb{R}_{+}$is coordinated geometrically convex on $\Delta$ for $a<b$ and $c<d$. Then

$$
\begin{aligned}
h(\sqrt{a b}, \sqrt{c d}) \leq \frac{1}{(\ln b-\ln a)(\ln d-\ln c)} & \int_{a}^{b} \int_{c}^{d} \frac{h(x, y)}{x y} \mathrm{~d} y \mathrm{~d} x \\
\leq & \frac{h(a, c)+h(a, d)+h(b, c)+h(b, d)}{4} .
\end{aligned}
$$

Theorem 8. Suppose that $h: \Delta=[a, b] \times[c, d] \subseteq \mathbb{R}^{2} \rightarrow \mathbb{R}_{+}$is logarithmically convex on coordinates $\Delta$ for $a<b$ and $c<d$. Then

$$
h\left(\frac{a+b}{2}, \frac{c+d}{2}\right) \leq \frac{1}{(b-a)(d-c)} \int_{a}^{b} \int_{c}^{d} h(x, y) \mathrm{d} y \mathrm{~d} x .
$$

Proof. Since $h$ is geometrically convex on coordinates $\Delta$, we have

$$
\begin{aligned}
& h\left(\frac{a+b}{2}, \frac{c+d}{2}\right)=\int_{0}^{1} \int_{0}^{1} h\left(\frac{t a+(1-t) b+(1-t) a+t b}{2},\right. \\
\left.\frac{\lambda c+(1-\lambda) d+(1-\lambda) c+\lambda d}{2}\right) \mathrm{d} t \mathrm{~d} \lambda & \int_{0}^{1} \int_{0}^{1}[h(t a+(1-t) b, \lambda c+(1-\lambda) d) h(t a+(1-t) b,(1-\lambda) c+\lambda d) \\
& \times h((1-t) a+t b, \lambda c+(1-\lambda) d) h((1-t) a+t b,(1-\lambda) c+\lambda d)]^{1 / 4} \mathrm{~d} t \mathrm{~d} \lambda \\
\leq & \frac{1}{4} \int_{0}^{1} \int_{0}^{1}[h(t a+(1-t) b, \lambda c+(1-\lambda) d)+h(t a+(1-t) b,(1-\lambda) c+\lambda d) \\
& +h((1-t) a+t b, \lambda c+(1-\lambda) d)+h((1-t) a+t b,(1-\lambda) c+\lambda d)] \mathrm{d} t \mathrm{~d} \lambda \\
= & \frac{1}{(b-a)(d-c)} \int_{a}^{b} \int_{c}^{d} h(x, y) \mathrm{d} y \mathrm{~d} x .
\end{aligned}
$$

Theorem 8 is thus proved.

Corollary 2. Under conditions of Theorems 4 and 8 , we have

$h\left(\frac{a+b}{2}, \frac{c+d}{2}\right) \leq \frac{1}{(b-a)(d-c)} \int_{a}^{b} \int_{c}^{d} h(x, y) \mathrm{d} y \mathrm{~d} x$ 


$$
\leq M_{h}(\Delta) \leq \frac{h(a, c)+h(a, d)+h(b, c)+h(b, d)}{4} .
$$

where $M_{h}(\Delta)$ is defined as in (1.3).

\section{Acknowledgements}

This work was partially supported by the National Natural Science Foundation of China (Grant No. 11361038), by the Foundation of the Research Program of Science and Technology at Universities of Inner Mongolia Autonomous Region (Grant No. NJZZ18154), and by the Inner Mongolia Autonomous Region Natural Science Foundation Project (Grant No. 2018LH01002), China.

The authors appreciate anonymous referees for their careful corrections to and valuable comments on the original version of this paper.

\section{REFERENCES}

[1] M. Alomari and M. Darus, "On the Hadamard's inequality for log-convex functions on the coordinates," J. Inequal. Appl., vol. 2009, p. Art. ID 28314713 pages, 2009, doi: $10.1155 / 2009 / 283147$.

[2] M. Avriel, "r-convex functions," Math. Programming, vol. 2, pp. 309-323, 1972, doi: 10.1007/BF01584551.

[3] M. Avriel, "Solution of certain nonlinear programs involving $r$-convex functions," J. Optimization Theory Appl., vol. 11, pp. 159-174, 1973, doi: 10.1007/BF00935881.

[4] R.-F. Bai, F. Qi, and B.-Y. Xi, "Hermite-Hadamard type inequalities for the $m$ - and $(\alpha, m)$-logarithmically convex functions," Filomat, vol. 27, no. 1, pp. 1-7, 2013, doi: 10.2298/FIL1301001B.

[5] S. S. Dragomir, "On the Hadamard's inequality for convex functions on the co-ordinates in a rectangle from the plane," Taiwanese J. Math., vol. 5, no. 4, pp. 775-788, 2001, doi: $10.11650 /$ twjm/1500574995.

[6] S. S. Dragomir and R. P. Agarwal, "Two inequalities for differentiable mappings and applications to special means of real numbers and to trapezoidal formula," Appl. Math. Lett., vol. 11, no. 5, pp. 91-95, 1998, doi: 10.1016/S0893-9659(98)00086-X.

[7] S. S. Dragomir and C. E. M. Pearce, "Selected topics on hermite-hadamard type inequalities and applications," RGMIA Monographs, p. 361 pages, 2000.

[8] P. M. Gill, C. E. M. Pearce, and J. Pečarić, "Hadamard's inequality for $r$-convex functions," $J$. Math. Anal. Appl., vol. 215, no. 2, pp. 461-470, 1997, doi: 10.1006/jmaa.1997.5645.

[9] B.-N. Guo and F. Qi, "A simple proof of logarithmic convexity of extended mean values," Numer. Algorithms, vol. 52, no. 1, pp. 89-92, 2009, doi: 10.1007/s11075-008-9259-7.

[10] B.-N. Guo and F. Qi, "The function $\left(b^{x}-a^{x}\right) / x$ : logarithmic convexity and applications to extended mean values," Filomat, vol. 25, no. 4, pp. 63-73, 2011, doi: 10.2298/FIL1104063G.

[11] N. P. N. Ngoc, N. V. Vinh, and P. T. T. Hien, "Integral inequalities of Hadamard type for $r$-convex functions," Int. Math. Forum, vol. 4, no. 33-36, pp. 1723-1728, 2009.

[12] C. P. Niculescu and L.-E. Persson, Convex Functions and Their Applications-A Contemporary Approach, 2nd ed., ser. CMS Books in Mathematics/Ouvrages de Mathématiques de la SMC. Springer, Cham, 2018. doi: 10.1007/978-3-319-78337-6.

[13] M. E. Özdemir and A. O. Akdemir, "On hadamard-type inequalities for co-ordinated $r$-convex functions," arXiv Preprint, p. 10 pages, 2010. 
[14] C. E. M. Pearce, J. Pečarić, and V. Šimić, "Stolarsky means and Hadamard's inequality,” J. Math. Anal. Appl., vol. 220, no. 1, pp. 99-109, 1998, doi: 10.1006/jmaa.1997.5822.

[15] F. Qi, "Logarithmic convexity of extended mean values," Proc. Amer. Math. Soc., vol. 130, no. 6, pp. 1787-1796, 2002, doi: 10.1090/S0002-9939-01-06275-X.

[16] F. Qi, “A note on Schur-convexity of extended mean values," Rocky Mountain J. Math., vol. 35, no. 5, pp. 1787-1793, 2005, doi: 10.1216/rmjm/1181069663.

[17] F. Qi, D. Lim, and B.-N. Guo, "Explicit formulas and identities for the Bell polynomials and a sequence of polynomials applied to differential equations," Rev. R. Acad. Cienc. Exactas Fís. Nat. Ser. A Mat. RACSAM, vol. 113, no. 1, pp. 1-9, 2019, doi: 10.1007/s13398-017-0427-2.

[18] F. Qi, D.-W. Niu, and B.-N. Guo, "Some identities for a sequence of unnamed polynomials connected with the Bell polynomials," Rev. R. Acad. Cienc. Exactas Fís. Nat. Ser. A Mat. RACSAM, vol. 113, no. 2, pp. 557-567, 2019, doi: 10.1007/s13398-018-0494-z.

[19] F. Qi, J. Sándor, S. S. Dragomir, and A. Sofo, "Notes on the Schur-convexity of the extended mean values," Taiwanese J. Math., vol. 9, no. 3, pp. 411-420, 2005, doi: 10.11650/twjm/1500407849.

[20] H.-N. Shi, S.-H. Wu, and F. Qi, "An alternative note on the Schur-convexity of the extended mean values," Math. Inequal. Appl., vol. 9, no. 2, pp. 219-224, 2006, doi: 10.7153/mia-09-22.

[21] M. Sun and X. Yang, "Inequalities for the weighted mean of $r$-convex functions," Proc. Amer. Math. Soc., vol. 133, no. 6, pp. 1639-1646, 2005, doi: 10.1090/S0002-9939-05-07835-4.

[22] S. H. Wu, " $r_{P}$-convex function and Jensen type inequality," Math. Practice Theory, vol. 35, no. 3, pp. 220-228, 2005.

[23] B.-Y. Xi, R.-F. Bai, and F. Qi, "Hermite-Hadamard type inequalities for the $m$ - and $(\alpha, m)$ geometrically convex functions," Aequationes Math., vol. 84, no. 3, pp. 261-269, 2012, doi: 10.1007/s00010-011-0114-X.

[24] B. Y. Xi and T. Y. Bao, "Some properties of $r$-mean convex functions," Math. Pract. Theory, vol. 38, no. 12, pp. 113-119, 2008.

[25] B.-Y. Xi, D.-D. Gao, T. Zhang, B.-N. Guo, and F. Qi, "Shannon type inequalities for Kapur's entropy," Mathematics, vol. 7, no. 1, pp. Article 2, 8 pages, 2019, doi: 10.3390/math7010022.

[26] B.-Y. Xi and F. Qi, "Some Hermite-Hadamard type inequalities for differentiable convex functions and applications," Hacet. J. Math. Stat., vol. 42, no. 3, pp. 243-257, 2013.

[27] B.-Y. Xi and F. Qi, "Hermite-Hadamard type inequalities for geometrically $r$-convex functions," Studia Sci. Math. Hungar, vol. 51, no. 4, pp. 530-546, 2014, doi: 10.1556/SScMath.51.2014.4.1294.

[28] B.-Y. Xi and F. Qi, "Inequalities of Hermite-Hadamard type for extended $s$-convex functions and applications to means," J. Nonlinear Convex Anal., vol. 16, no. 5, pp. 873-890, 2015.

[29] G.-S. Yang and D.-Y. Hwang, "Refinements of Hadamard's inequality for $r$-convex functions," Indian J. Pure Appl. Math., vol. 32, no. 10, pp. 1571-1579, 2001.

[30] G. Zabandan, A. Bodaghi, and A. Kı lıçman, "The Hermite-Hadamard inequality for $r$-convex functions," J. Inequal. Appl., vol. 2012, pp. Article 215, 8 pages, 2012, doi: 10.1186/1029-242X2012-215.

Authors' addresses

Dan-Dan Gao

College of Mathematics, Inner Mongolia University for Nationalities, Tongliao 028043, China

E-mail address: Dan-DanGao@hotmail.com

Bo-Yan Xi

College of Mathematics, Inner Mongolia University for Nationalities, Tongliao 028043, China

E-mail address: baoyintu78@qq.com 
Ying Wu

College of Mathematics, Inner Mongolia University for Nationalities, Tongliao 028043, China

E-mail address: wuying19800920@qq.com

Bai-Ni Guo

(Corresponding author) School of Mathematics and Informatics, Henan Polytechnic University, Jiaozuo 454010, China

E-mail address: bai.ni.guoegmail.com 